Jandira Pereira dos Santos ${ }^{1}$

Airton Pozo de Mattos ${ }^{2}$

\section{Absentismo-doença na prefeitura municipal de Porto Alegre, Rio Grande do Sul, Brasil*}

\author{
Ilness absenteeism in the municipal administration of Porto \\ Alegre, Brazil
}

\begin{abstract}
${ }^{1}$ Mestre em Saúde Coletiva pela Universidade Luterana do Brasil (ULBRA), Canoas, RS.

${ }^{2}$ Docente do Programa de Pós-Graduação em Saúde Coletiva, Universidade Luterana do Brasil (ULBRA), Canoas, RS.

*Trabalho apresentado no II Congresso Catarinense de Saúde Coletiva, realizado em Florianópolis, SC, de 11 a 14 de novembro de 2008; e no 9o Congresso de Stress da ISMA-BR (International Stress Management Association no Brasil), $11^{\circ}$ Fórum Internacional de Qualidade de Vida no Trabalho, 1 을 Encontro de Qualidade de Vida na Segurança Pública e 1ํㅡㄹ Encontro de Qualidade de Vida no Serviço Público, realizado em Porto Alegre, $\mathrm{RS}$, de 23 a 25 de junho de 2009.

Artigo baseado na dissertação de mestrado de Jandira Pereira dos Santos intitulada Absentismo-doença em uma prefeitura municipal do sul do Brasil, apresentada em 2008 ao Programa de Pós-Graduação em Saúde Coletiva da Universidade Luterana do Brasil (ULBRA).
\end{abstract}

\section{Contato:}

Jandira Pereira dos Santos Rua Ingá, 60, Parque Florestal - Itapuã CEP: 94 750-000 - Viamão, RS

E-mail:

jandirapsantos@yahoo.com.br
}

Recebido: 29/05/2009

Revisado: 08/12/2009

Aprovado: 17/12/2009

\section{Resumo}

Objetivo: O absentismo-doença é o principal motivo de afastamentos de servidores na Prefeitura de Porto Alegre, RS, Brasil. O objetivo deste estudo foi analisar as licenças para tratamento de saúde (LTS) concedidas a servidores da Prefeitura. Metodologia: As informações foram fornecidas pela Gerência de Saúde do Servidor Municipal (GSSM). Foram analisadas LTS $\geq 15$ dias concedidas aos servidores no período de 1 o de janeiro de 2004 a 31 de dezembro de 2005. Resultados: As LTS $\geq 15$ dias concedidas no período somaram 14.779. Em 2004, foram concedidas 6.522 licenças a 1.963 servidores, a uma razão de 3,3 LTS por servidor; em 2005, a concessão foi de 8.257 LTS para 2.262 servidores, a uma razão de 3,6. Considerando uma significância de $5 \%$, foi observada uma diferença entre as proporções de licenças $\geq 15$ dias concedidas em 2004 e em 2005. No período, foram perdidos 311.721 dias de trabalho. A taxa de absentismo-doença foi de 3,9\% em 2004 e de 3,7\% em 2005. Os transtornos mentais foram as doenças que mais afastaram os servidores, com uma proporção de 39,59\%. A secretaria com maior índice de absentismo-doença no período foi a Secretaria Municipal de Esportes. Conclusão: O conhecimento destes dados poderá permitir uma ação mais efetiva na prevenção e no controle do absentismo-doença com o intuito de diminuir as taxas encontradas.

Palavras-chave: absentismo-doença; saúde do trabalhador; servidor público; administração pública; saúde ocupacional.

\section{Abstract}

Objective: Absenteeism among civil servants of the municipality of Porto Alegre, southern Brazil, is mainly due to illness. The objective of this study was to assess sick leaves (SL) among municipal workers. Methodology: Information regarding $S L$ was collected at the Health Management for Municipal Servants in the Municipal Department of Health in Porto Alegre. SL $\geq 15$ days granted between January 1, 2004 and December 31, 2005 were analyzed. Results: A total of $14,779 S L \geq 15$ days were granted in the period. In 2004, 1,963 workers received 6,522 SL, at a ratio of 3.3 SL per worker; in 2005, 2,262 workers were granted 8,257 SL, at a ratio of 3.6. Considering a significance of 5\%, a difference was observed between the ratios of $S L \geq 15$ days granted in 2004 and in 2005. The number of lost work days amounted to 311,721. The rate of illness absenteeism among civil servants from the city administration of Porto Alegre was 3,9\% in 2004 and $3.7 \%$ in 2005. Mental and behavioral disorders were the main causes for absenteeism (39.59\%). The department with the highest sick leave rate in the period was the Sports Department. Conclusion: Knowledge of these data may contribute to more effective action in preventing and managing absenteeism, so as to decrease the observed rates.

Keywords: illness absenteeism; worker's health; civil servants; public management; occupational health. 


\section{Introdução}

Saúde e trabalho são temas relacionados entre si: ambos fazem parte do ambiente no qual o trabalhador exerce sua atividade e passa a maior parte de sua vida. O trabalho ocupa um lugar importante na vida de qualquer pessoa e é através dele que o homem busca seu sustento, satisfação, valorização e realização como ser humano útil e provido de capacidade intelectual (DEJOURS, 1986; GUIMARÃES, 2005; FARIA; BARBOZA; DOMINGOS, 2005). A dinâmica dessas relações, o ritmo acelerado, a competitividade e a jornada excessiva de trabalho contribuem para a baixa autoestima, fadiga, estresse e adoecimento do trabalhador (ABRAMIDES; CABRAL, 2003).

Mendes (1988) chama a atenção para a relação trabalho/ocupação como causa de doença. O mesmo autor afirma que a ocorrência dessas doenças em função do trabalho, seja pelo ambiente, pelo processo de produção ou, ainda, pela organização do trabalho, é evitável e propõe o envolvimento do setor de saúde no sentido de intervir para prevenir e controlar as causas das doenças relacionadas ao trabalho que podem levar o trabalhador à morte.

Os trabalhadores públicos, estatutários, civis e militares, denominados na Constituição de 1988 como "servidores”, somavam 5,9 milhões em 2006 (INSTITUTO BRASILEIRO DE GEOGRAFIA E ESTATISTICA, 2007). No Brasil, a categoria de servidores públicos é um segmento social pouco conhecido, seja em nível municipal, estadual ou federal. Essa categoria forma um contingente de trabalhadores que atua diretamente na elaboração de projetos ou na execução de tarefas, que interferem de imediato na qualidade de vida do povo (DURAND; BELTRÃO, 1994).

França (1994) refere que o servidor é um trabalhador como outro qualquer e que a condição de trabalho do funcionário público em nada se diferencia da classe operária brasileira. A autora afirma que o suposto "privilégio" do servidor público, que muitos propagam, está restrito à estabilidade e ao fato de estar empregado.

O absentismo ${ }^{2}$ ocupacional é um grande problema das organizações, sejam elas públicas ou privadas (DOMINGUES JÚNIOR, 2005). Caracteriza-se por falta ou ausência constante do empregado ao trabalho, falta de assiduidade, atraso por qualquer motivo, férias ou folgas (TENÓRIO; JORDANO, 1980; MALLADA, 2004; HURREL, 1998). Embora o absentismo ocupacional se deva a inúmeros fatores, o que mais afasta o trabalhador é o absentismo-doença.

O absentismo-doença está relacionado à incapacidade temporária, prolongada ou permanente, para trabalhar, resultante de doença ou indisposição. O trabalhador fica impedido de comparecer ao trabalho em função de alguma doença ou acidente de ou no trabalho, normalmente comprovado mediante atestado médico (HURREL, 1998; FUNDACIÓN EUROPEA PARA LA MEJORA DE LAS CONDICIONES DE VIDA Y TRABAJO, 1997).

De acordo com dados levantados pela Câmara de Vereadores, através da Comissão Parlamentar de Inquérito (CPI) do Alto Índice de Afastamento de Servidores Municipais por Biometria (CÂMARA DOS VEREADORES DE PORTO ALEGRE, 2006a), os índices de licenças para o tratamento de saúde (LTS) entre os trabalhadores municipais na Prefeitura Municipal de Porto Alegre são elevados. Assim, o objetivo da presente investigação foi estudar as LTS $\geq 15$ dias, concedidas no período de $1^{\underline{0}}$ de janeiro de 2004 a 31 de dezembro de 2005.

\section{Materiais e métodos}

Este é um estudo epidemiológico transversal descritivo, realizado a partir de dados secundários fornecidos pela Gerência de Saúde do Servidor Municipal (GSSM). Para a análise dos dados, foram seguidas as recomendações do Subcomitê de Absentismo da Associação Internacional de Medicina do Trabalho (QUICK; LAPERTOSA, 1982; GOMERO CUADRA; LLAP YESAN, 2004; COUTO, 1987; DANATRO, 1997; SILVA; MARZIALE, 2000). Os dados fornecidos foram organizados em planilha do Excel e exportados para o programa de análise estatística Statistical Package for the Social Sciences (SPSS), versão 12.

Foram estudadas LTS concedidas a servidores da Prefeitura de Porto Alegre no período de $1^{\circ}$ de janeiro de 2004 a 31 de dezembro de 2005 com duração $\geq 15$ dias.

É importante observar que alguns dos departamentos incluídos nos dados de 2005 não constavam dos dados informados para 2004, por exemplo, Departamento $\mathrm{Mu}$ nicipal de Limpeza Urbana (DMLU), Departamento Municipal de Habitação (DEMHAB), Departamento Municipal de Água e Esgoto (DMAE), entre outros. Diante disso, nem todas as LTS $\geq 15$ dias da Prefeitura de Porto Alegre foram analisadas. A Companhia Carris Porto-Alegrense (CARRIS), a Empresa Pública de Transporte e Circulação (EPTC) e o Departamento Municipal de Previdência dos Servidores Públicos do Município (PREVIMPA) não constaram tanto em 2004, como em 2005. Além disso, nos dados fornecidos para o ano de 2004, não havia registro das doenças que deram origem às licenças, portanto, não foi possível verificar quais doenças afastaram os servidores.

Trabalhou-se com 248 dias úteis. Foram excluídos 104 dias, entre domingos, sábados e 13 feriados. As fórmulas utilizadas para calcular a taxa de absentismo-doença (Tad), o índice de frequência (If), o índice de gravidade (Ig) e a duração média das licenças (Dml) estão descritas abaixo:

\footnotetext{
${ }^{2}$ Quick e Lapertosa (1982) referem que o termo “absenteísmo” é usado como sinônimo de "absentismo”. Os autores afirmam que absenteísmo significa "abstencionismo", "abstenção ao exercício do voto". Neste estudo, usaremos o termo "absentismo" por entendermos ser este o termo correto.
} 


$$
\begin{aligned}
& \text { Tad }=\left(\frac{\text { total de dias de licença médica }}{\mathrm{n}^{\circ} \text { de servidores } \times \mathrm{n}^{\circ} \text { de dias úteis }}\right) \times 100 \\
& I g=\frac{\mathrm{n}^{\circ} \text { de dias de afastamento total }}{\mathrm{n}^{\circ} \text { de servidores }}
\end{aligned}
$$

Foi calculada a razão de licenças por servidor no período, e considerada uma significância de $5 \%$ para verificar a presença de diferença significativa entre as proporções.

A pesquisa atendeu à Resolução no 196, de 10 de outubro de 1996, do Conselho Nacional de Saúde/BR, que normatiza a pesquisa com seres humanos. O presente estudo foi aprovado pelo Comitê de Ética e Pesquisa da Secretaria Municipal de Saúde de Porto Alegre, RS.

\section{Resultados}

Foi possível observar que, no período estudado, foram concedidas licenças não motivadas por questões médicas. $\mathrm{O}$ acidente de trabalho, que não é um tipo de licença, chama a atenção, pois deu origem a 2.206 LTS no período do estudo. Estes casos não foram considerados na análise dos resultados.

$$
\begin{gathered}
\text { If }=\left(\frac{\mathrm{n}^{\mathrm{o}} \text { de licenças médicas }}{\mathrm{n}^{\mathrm{o}} \text { de servidores }}\right) \times 100 \\
D m l=\frac{\mathrm{n}^{\mathrm{o}} \text { de dias de afastamento total }}{\mathrm{n}^{\circ} \text { de licenças médicas }}
\end{gathered}
$$

As licenças por motivo de doenças e de menor duração somaram 44.804 e merecem ser investigadas em outro estudo.

Em 2004, as LTS $\geq 15$ dias foram concedidas a 1.963 servidores, somando 6.522 licenças, uma razão de 3,3 LTS por servidor. Em 2005, as LTS concedidas a 2.262 servidores somaram 8.257 licenças a uma razão de 3,6 licenças por servidor.

Considerando uma significância de 5\%, foi possível observar uma diferença entre as proporções de licenças $\geq 15$ dias concedidas em 2004 e em 2005. O total de dias de afastamento no período foi de 311.713 dias.

Na Tabela 1, observa-se a Tad por secretaria em 2004. A Secretaria Municipal de Esportes (SME) foi o órgão com a maior Tad (5,5\%), seguida da Secretaria Municipal de Cultura (SMC), com 5,2\%. Das secreta-

Tabela 1 Indicadores de absentismo-doença considerando afastamentos $\geq 15$ dias, na Prefeitura Municipal de Porto Alegre, RS, em 2004

\begin{tabular}{lccccccc}
\hline \multicolumn{1}{c}{ Órgão $^{1}$} & LTS & $N^{2}$ de servidores & $I f^{3}$ & NDAFTOT $^{4}$ & $I^{5}$ & DML $^{6}$ & Tad $^{7}$ \\
\hline SME & 98 & 163 & 60,1 & 2230 & 13,6 & 22,7 & 5,5 \\
SMC & 161 & 261 & 61,6 & 3414 & 13,0 & 21,2 & 5,2 \\
SMED & 2816 & 5060 & 55,6 & 60442 & 11,9 & 21,4 & 4,8 \\
SMOV & 483 & 883 & 54,6 & 10464 & 11,8 & 21,6 & 4,7 \\
SMAM & 364 & 678 & 53,6 & 7826 & 11,5 & 21,5 & 4,6 \\
SMIC & 126 & 290 & 43,4 & 2685 & 9,2 & 21,3 & 3,7 \\
SMT & 63 & 151 & 41,7 & 1399 & 9,2 & 22,2 & 3,7 \\
SMS & 1538 & 3928 & 39,1 & 33342 & 8,4 & 21,6 & 3,4 \\
DEP & 94 & 269 & 34,9 & 2030 & 7,5 & 21,5 & 3,0 \\
FASC & 178 & 551 & 32,3 & 3643 & 6,6 & 20,4 & 2,6 \\
PGM & 36 & 94 & 38,2 & 734 & 7,8 & 20,3 & 3,1 \\
SMF & 126 & 402 & 31,3 & 2674 & 6,6 & 21,2 & 2,6 \\
SMA & 104 & 349 & 29,7 & 2088 & 5,9 & 20,0 & 2,4 \\
SPM & 41 & 163 & 25,1 & 905 & 5,5 & 22,0 & 2,2 \\
SMDHSU & 152 & 609 & 24,9 & 3337 & 5,4 & 21,9 & 2,2 \\
GP & 22 & 107 & 20,5 & 469 & 4,3 & 21,3 & 1,7 \\
Outros Órgãos & 120 & 150 & 0,8 & 131 & 0,8 & 1,09 & 0,3 \\
\hline Total & 6522 & 14108 & 46,2 & 137813 & 9,7 & 21,1 & 3,9 \\
\hline
\end{tabular}

'Órgão: DEP: Departamento de Esgotos Pluviais; FASC: Fundação de Assistência Social e Cidadania; GP: Gabinete do Prefeito; PGM: Procuradoria Geral do Município; SMA: Secretaria Municipal de Administração; SMAM: Secretaria Municipal do Meio Ambiente; SMC: Secretaria Municipal de Cultura; SMDHSU: Secretaria Municipal de Direitos Humanos e Segurança Urbana; SME: Secretaria Municipal de Esportes; SMED: Secretaria Municipal de Educação; SMF: Secretaria Municipal da Fazenda; SMIC: Secretaria Municipal da Indústria e Comércio; SMOV: Secretaria Municipal de Obras e Viação; SMS: Secretaria Municipal da Saúde; SPM: Secretaria de Planejamento Municipal; SMT: Secretaria Municipal do Turismo;

${ }^{2}$ LTS: licença para tratamento de saúde;

${ }^{3}$ If: índice de frequência;

${ }^{4}$ NDAFTOT: número de dias de afastamento total;

${ }^{5}$ Ig: índice de gravidade;

${ }^{6}$ DML: duração média das licenças;

${ }^{7}$ Tad: taxa de absentismo-doença. 
rias denominadas operacionais, conforme relatório da CPI das Biometrias, a que possui o maior índice de absentismo-doença é a Secretaria Municipal de Educação (SMED) com 4,8\%, seguida pela Secretaria Municipal de Obras e Viação (SMOV) com 4,7\%. A Secretaria Municipal da Saúde (SMS) ocupa o oitavo lugar, com índice de absentismo-doença de 3,4\%. O Gabinete do Prefeito (GP) é o órgão com o menor índice de absentismo-doença na Prefeitura em 2004.

A Tad na Prefeitura, tendo como base o número de servidores das secretarias estudadas em 2004 (14.108) e em função das LTS $\geq 15$ dias, foi de $3,9 \%$. O If de LTS foi de $46,2 \%$. O número de dias de afastamento total, em 2004, foi de 137.813, o Ig foi de 9,7 e a DML foi de 21,1 dias.

Na Tabela 2, observa-se a Tad por secretaria no ano de 2005. A SME continua a apresentar a maior taxa, porém com um percentual menor em relação a $2004(5,0 \%)$. A SMED ocupa o segundo lugar em 2005, apresentando uma Tad de 4,8\%, índice maior quando comparado a 2004. A SMC fica em terceiro lugar, com taxa de 4,7\%, menor quando comparada à taxa de 2004. A SMS ocupa o oitavo lugar, com índice de absentismo-doença de 3,9\%, maior quando comparado com 2004. A Secretaria Municipal de Coordenação Política e Governança Local (SMCPGL), em 2005, foi a secretaria com o menor índice de absentismo-doença $(0,7 \%)$.

A Tad na Prefeitura, tendo como base o número de servidores das secretarias estudadas em 2005 (18.579) e em função das LTS $\geq 15$ dias, foi de $3,7 \%$. O If de LTS foi de 44,4\%. O número de dias de afastamento total, em 2005, foi de 173.908, o Ig foi de 9,3 e a DML foi de 21,0 dias.

Tabela 2 Indicadores de absentismo-doença por secretaria na Prefeitura Municipal de Porto Alegre, RS, em 2005, considerando afastamentos $\geq 15$ dias

\begin{tabular}{lccccccc}
\hline \multicolumn{1}{c}{ Órgão $^{1}$} & LTS & № de servidores & \multicolumn{1}{c}{$\beta^{3}$} & NDAFTOT $^{4}$ & $I^{5}$ & DML $^{6}$ & Tad $^{7}$ \\
\hline SME & 84 & 157 & 53,5 & 1959 & 12,4 & 23,3 & 5,0 \\
SMED & 2944 & 5050 & 58,2 & 60685 & 12,0 & 20,6 & 4,8 \\
SMC & 131 & 252 & 51,9 & 2949 & 11,7 & 22,5 & 4,7 \\
SMT & 68 & 150 & 45,3 & 1679 & 11,1 & 24,6 & 4,5 \\
DEMHAB & 202 & 439 & 46,0 & 4751 & 10,8 & 23,5 & 4,3 \\
DMLU & 826 & 1729 & 47,7 & 17948 & 10,3 & 21,7 & 4,1 \\
SMAM & 327 & 657 & 49,7 & 7005 & 10,6 & 21,4 & 4,2 \\
SMS & 1753 & 3831 & 45,7 & 37193 & 9,7 & 21,2 & 3,9 \\
SMOV & 348 & 854 & 40,7 & 7155 & 8,3 & 20,5 & 3,3 \\
SMIC & 105 & 283 & 37,1 & 2090 & 7,3 & 19,9 & 2,9 \\
FASC & 202 & 547 & 36,9 & 4174 & 7,6 & 20,6 & 3,0 \\
SPM & 57 & 158 & 36,0 & 1154 & 7,3 & 20,2 & 2,9 \\
SMDHSU & 175 & 590 & 29,6 & 3835 & 6,5 & 21,9 & 2,6 \\
PGM & 33 & 94 & 35,1 & 720 & 7,6 & 21,8 & 3,0 \\
SMA & 100 & 343 & 29,1 & 2023 & 5,8 & 20,2 & 2,3 \\
DMAE & 634 & 2383 & 26,6 & 13825 & 5,8 & 21,8 & 2,3 \\
SMF & 103 & 402 & 25,6 & 2312 & 5,7 & 22,4 & 2,3 \\
DEP & 70 & 268 & 26,1 & 1419 & 5,2 & 20,2 & 2,1 \\
SMGAE & 13 & 100 & 13,0 & 268 & 2,6 & 20,6 & 1,0 \\
Outros órgãos & 62 & 150 & 41,3 & 396 & 2,6 & 6,3 & 1,0 \\
GP & 11 & 51 & 21,5 & 202 & 3,9 & 18,3 & 1,5 \\
SMCPGL & 09 & 91 & 9,8 & 166 & 1,8 & 18,4 & 0,7 \\
\hline Total & 8257 & 18579 & 44,4 & 173908 & 9,3 & 21,0 & 3,7 \\
\hline
\end{tabular}

1 Órgão: DEMHAB: Departamento Municipal de Habitação; DEP: Departamento de Esgotos Pluviais; DMAE: Departamento Municipal de Água e Esgoto; DMLU: Departamento Municipal de Limpeza Urbana; FASC: Fundação de Assistência Social e Cidadania; GP: Gabinete do Prefeito; PGM: Procuradoria Geral do Município; SMA: Secretaria Municipal de Administração; SMAM: Secretaria Municipal do Meio Ambiente; SMC: Secretaria Municipal de Cultura; SMCGL: Secretaria Municipal de Coordenação Política e Governança Local; SMDHSU: Secretaria Municipal de Direitos Humanos e Segurança Urbana; SMGAE: Secretaria Municipal de Gestão e Acompanhamento Estratégico; SME: Secretaria Municipal de Esportes; SMED: Secretaria Municipal de Educação; SMF: Secretaria Municipal da Fazenda; SMIC: Secretaria Municipal da Indústria e Comércio; SMOV: Secretaria Municipal de Obras e Viação; SMS: Secretaria Municipal da Saúde; SPM: Secretaria de Planejamento Municipal; SMT: Secretaria Municipal do Turismo;

${ }^{2}$ LTS: licença para tratamento de saúde;

${ }^{3}$ If: índice de frequência;

${ }^{4}$ NDAFTOT: número de dias de afastamento total;

${ }^{5}$ Ig: índice de gravidade;

${ }^{6}$ DML: duração média das licenças;

${ }^{7}$ Tad: taxa de absentismo-doença. 
O DEMHAB é a autarquia que apresenta a maior Tad, com $4,3 \%$, seguido pelo DMLU, com $4,1 \%$, e pelo DMAE, com 2,3\%. O Departamento de Esgotos Pluviais (DEP) é o departamento com a menor Tad $(2,1 \%)$.

Na Tabela 3, é possível observar as LTS por categorias de doenças, de acordo com o Código Internacional de Doenças (CID-10).

Dentre as doenças que mais afastaram os servidores, as de maiores proporções $(39,59 \%)$ estiveram relacionadas aos transtornos mentais e comportamentais (F00-F99). Nesta categoria, verificou-se que os diagnósticos mais frequentes foram: episódios depressivos $(\mathrm{F} 32=12,5 \%)$, transtorno afetivo bipolar (F31 = $8,1 \%$ ), episódio depressivo grave sem sintomas psicóticos (F32.2 = 7,7\%), transtorno depressivo recorrente (F33 $=7,6 \%)$ e transtornos mentais e comportamentais causados pelo uso de álcool (F10 = 4,7\%).
Para as licenças vinculadas à categoria "fatores que influenciam o estado de saúde e o contato com os serviços de saúde" (Z00-Z99), os diagnósticos mais frequentemente observados foram: convalescença após cirurgia $(Z 54.0=41,9 \%)$, convalescença $(Z 54=33,2 \%)$ e exame médico e consulta com finalidades administrativas (Z02 $=11,8 \%$ ). No CID-10, esta categoria é utilizada quando não existe uma doença, trauma ou causa externa:

a pessoa, que não está doente, procura o serviço de
saúde para receber assistência ou serviço limitado para
uma afecção atual, doar órgão ou tecido, receber imu-
nização profilática ou discutir um problema que não é
em si uma doença ou um traumatismo. (CID-10)

Para as licenças vinculadas à categoria "doenças do sistema osteomuscular e do tecido conjuntivo" (M00M99), verificou-se que os diagnósticos mais frequentes foram: dorsalgia (M54 = 16,1\%), sinovite e tenossinovite $(\mathrm{M} 65=11,0 \%)$ e cervicalgia (M54.2 = 5,4\%).

Tabela 3 Distribuição dos diagnósticos (segundo CID-10) mais envolvidos nas licenças para tratamento de saúde em servidores da Prefeitura Municipal de Porto Alegre, RS, em 2005

\begin{tabular}{|c|c|c|}
\hline Categoria CID-10 1 & № de LTS 2 & $\%$ \\
\hline Transtornos mentais e comportamentais (F00-F99) & 3255 & 39,45 \\
\hline Fatores que influenciam o estado de saúde e o contato com os serviços de saúde (Z00-Z99) & 1266 & 15,34 \\
\hline Doenças do sistema osteomuscular e do tecido conjuntivo (M00-M99) & 820 & 9,93 \\
\hline Doenças do aparelho circulatório (100-199) & 390 & 4,73 \\
\hline Lesões, envenenamentos e algumas outras consequências de causas externas (S00-T98) & 369 & 4,47 \\
\hline Neoplasias [tumores] (C00-D48) & 272 & 3,30 \\
\hline Algumas doenças infecciosas e parasitárias (A00-B99) & 172 & 2,10 \\
\hline Gravidez, parto e puerpério (000-099) & 168 & 2,03 \\
\hline Doenças do aparelho respiratório (J00-J99) & 118 & 1,42 \\
\hline Doenças do aparelho digestivo (K00-K93) & 110 & 1,33 \\
\hline Doenças do sistema nervoso (G00-G99) & 108 & 1,30 \\
\hline Doenças do olho e anexos (H00-H59) & 79 & 0,95 \\
\hline Doenças do aparelho geniturinário (N00-N99) & 66 & 0,80 \\
\hline Doenças endócrinas, nutricionais e metabólicas (E00-E90) & 65 & 0,79 \\
\hline Doenças da pele e do tecido subcutâneo (L00-L99) & 26 & 0,31 \\
\hline CID Não Identificado & 973 & 11,7 \\
\hline Total & 8257 & 100,00 \\
\hline
\end{tabular}

${ }^{1}$ CID-10: Classificação Internacional de Doenças;

${ }^{2}$ LTS: licença para tratamento de saúde. 


\section{Discussão}

O presente estudo teve como objetivo analisar o absentismo-doença que afasta parte dos servidores da Prefeitura de Porto Alegre por 15 dias ou mais. No entanto, achamos importante evidenciar que outros tipos de licença responsáveis pelo afastamento de servidores atingiram um percentual de $28,6 \%$. O total de licenças no período de 1o de janeiro de 2004 a 31 de dezembro de 2005 foi de 72.487. O total de dias de afastamento foi de 694.457. A LTS foi a licença que mais afastou os servidores no período, totalizando 59.583 licenças, um percentual de $82,19 \%$.

$\mathrm{O}$ acidente de trabalho e o auxílio-doença foram registrados como tipos de licença. Tal registro, a nosso ver, é equivocado, pois, de acordo com a Lei Complementar no 133 (PORTO ALEGRE, 1985), que determina as relações da Prefeitura com seus servidores, o auxílio-doença e o acidente de trabalho não são tipos de licença. Entende-se que ambos os eventos são registrados de forma equivocada, não por lacuna da lei, mas possivelmente por descuido, falta de entendimento da própria lei ou simplesmente negligência em registrar. Nossa convicção de que se trata de um registro equivocado foi o que embasou a decisão de deixar esses 2.206 registros fora da análise estatística.

A nosso entender, o registro dos eventos citados deveria ser apontado como LTS, com o respectivo código CID da doença que ocasionou o afastamento.

$\mathrm{O}$ acidente de trabalho causa o adoecimento do servidor e provoca o seu afastamento (LTS). O auxílio-doença ocorre quando o servidor é afastado por 15 dias ou mais em função de uma determinada doença. A defesa para que se registre a LTS juntamente com o respectivo código CID é no sentido de saber quais são as doenças que mais acometem o servidor e que tipo de moléstia foi gerada pelo acidente de ou no trabalho.

Alguns questionamentos que necessitam de resposta, porém não neste estudo, são os seguintes: Qual a duração dos afastamentos que têm o acidente de trabalho como causa? O acidente ocasionou invalidez? Quais as secretarias que apresentam esse evento com maior frequência? Quais as medidas que estão sendo tomadas para o enfrentamento do acidente de ou no trabalho? Tais reflexões são relevantes especialmente ao se considerar que o acidente de trabalho foi responsável por 2.206 afastamentos no período do estudo.

Outro aspecto a ser considerado é a predominância das LTS de curta e média duração. Tal fato não é surpresa, pois a literatura consultada aponta que as faltas ao trabalho variam entre 2 e 14 dias de afastamento (GUIMARÃES, 2005; GOMERO CUADRA; LLAP YESAN, 2004; DANATRO, 1997; SILVA; MARZIALE, 2000; ISOSAKI, 2003; REIS et al., 2003; NOGUEIRA, 1980). Essa constatação requer um novo estudo com o objetivo de conhecer melhor o fenômeno do adoecimento entre os servidores e as implicações das frequentes ausências na organização do trabalho.
O período de 2004/2005, para a realização do estudo, foi escolhido previamente e de forma intencional em função de contemplar o último ano de um governo e o início de outra administração, com orientações políticas diferentes. Os objetivos eram claros: 1) descartar qualquer uso da pesquisa em debates de cunho político-partidário, sem resultados práticos para o conjunto de servidores; 2) apontar, para o gestor que iniciava, um pouco da realidade sobre a saúde do servidor, subsidiando a tomada de decisões; e, finalmente, 3) estimular pesquisas junto aos trabalhadores públicos em todos os níveis de governo. A escolha das licenças $\geq$ 15 dias foi em virtude do prejuízo que elas acarretam ao município no que diz respeito tanto ao numerário que o Instituto de Previdência deveria prover quando ocorre o afastamento, quanto ao adoecimento dos servidores que permanecem longos períodos afastados.

Para o controle e a prevenção do absentismo-doença, é preciso conhecer os fatores sociais, comportamentais e trabalhistas envolvidos, identificando-se a população-alvo para elaborar programas e propor estratégias de intervenção a fim de prevenir e controlar, de maneira eficaz, este fenômeno sociológico (MALLADA, 2004). São poucos os estudos relacionados à população de servidores públicos no Brasil (DURAND; BELTRÃ̃O, 1994). Já em instituições hospitalares, sejam elas públicas ou privadas, pesquisas referentes ao absentismo-doença são realizadas normalmente (GUIMARÃES, 2005; FARIA; BARBOZA; DOMINGOS, 2005; SILVA; MARZIALE, 2000; ISOSAKI, 2003; REIS et al., 2003; ECHER et al., 1999; SILVEIRA; MONTEIRO, 2004; BARBOSA; SOLER, 2003).

No período estudado, foram analisadas 14.779 licenças de longa duração, distribuídas nas diversas secretarias da Prefeitura de Porto Alegre. As LTS foram solicitadas por 4.225 servidores. O total de dias de trabalho perdidos no período foi de 311.721 , equivalendo a 1.256 anos de trabalho perdido ao se considerar 248 dias úteis. O número de trabalhadores que se afastaram por 15 dias ou mais representa 9,8\% da mão de obra da Prefeitura, que possui, no período estudado, 42.768 servidores (PREFEITURA MUNICIPAL DE PORTO ALEGRE, 2006). Contando férias, licenças-prêmio, nojo, gala, LTS de menor duração, doação de sangue, entre outros tipos de licenças e autorizações particulares, é possível afirmar que a Prefeitura funciona com um efetivo extremamente reduzido. Essa redução de servidores traz consequências diversas ao funcionamento administrativo, técnico e operacional e tal fato terá reflexo direto na qualidade dos serviços prestados à população.

Em alguns casos observados, os afastamentos chegam a ser de um ano ou mais. Alguns autores referem que afastamentos de longa duração sugerem problemas de saúde de maior gravidade (GUIMARÃES, 2005; FARIA; BARBOZA; DOMINGOS, 2005).

As organizações públicas apresentam maior quantidade de dias perdidos por absentismo-doença, bem como um período de afastamento maior do que as em- 
presas privadas (MARTINS et al., 2005; FARIA; BARBOZA; DOMINGOS, 2005). Tal fato pode ocorrer em função: da falta de critérios para a análise de atestados fornecidos por profissionais não vinculados à instituição, da carência de pessoal para o atendimento da demanda e da falta de um serviço médico de qualidade que acompanhe o servidor durante sua vida funcional.

A estabilidade no emprego é um fator que deve ser considerado ao se analisar o fenômeno do absentismo-doença entre servidores públicos estatutários, pois os trabalhadores se sentem mais seguros ao se afastarem do trabalho (REIS et al., 2003).

Neste estudo, assim como em outros (CÂMARA DOS VEREADORES DE PORTO ALEGRE, 2006a; DANATRO, 1997; SILVA; MARZIALE, 2000; PREFEITURA MUNICIPAL DE PORTO ALEGRE, 2006; PORTO ALEGRE, 1985; NOGUEIRA, 1980; BRASIL, 2001), foi observado que as LTS ocorreram em maior número nos meses que correspondem ao inverno, com predominância do mês de agosto. A ocorrência maior de licenças no inverno pode ser em função do frio, com a instalação de doenças que se agravam em função das baixas temperaturas.

\section{Taxas de absentismo-doença (Tad)}

As Tad de 3,9\% (2004) e 3,7\% (2005) encontradas neste estudo não permitem definir uma taxa geral de absentismo-doença para a Prefeitura de Porto Alegre por dois motivos: 1) não foram consideradas as LTS inferiores a 15 dias; 2) nos dados fornecidos pela GSSM, não constaram todas as secretarias, departamentos e empresas que compõem a Prefeitura, como, por exemplo, o PREVIMPA, entre outros. É possível que a Tad resultasse maior se fossem computadas todas as ausências decorrentes de licenças médicas.

As taxas de absentismo-doença encontradas no estudo são consideradas altas quando comparadas com o índice aceito pela Organização Internacional do Trabalho (OIT), que é de 2,5\%, porém, quando comparadas às taxas encontradas na literatura, são da mesma magnitude. É possível, portanto, que o índice aceito pela OIT esteja subestimado e fora da realidade e, por este motivo, podemos afirmar que os índices aqui encontrados estão de acordo com os encontrados na literatura (HURREL, 1998).

Mesmo que as taxas não tenham sido significativas quando relacionadas aos dados encontrados na literatura, elas devem servir como alerta aos gestores no que diz respeito à prevenção e à promoção da saúde do servidor municipal.

A SME, com Tad de 5,5\% em 2004 e de 5,0\% em 2005, foi a secretaria com os índices mais altos. Em 2004, a SME possuía 163 servidores e, em 2005, 157. Com uma frequência de 60,1\% em 2004 e 53,5\% em 2005 (prevalência de LTS nesta secretaria), não é possível definir como este órgão dá conta das demandas de sua competência. As secretarias congregadas pela prefeitura são comparáveis às mencionadas em outros estudos na literatura. Assim, os dados obtidos nesta investigação poderão servir como parâmetro para estudos posteriores sobre absentismo-doença na Prefeitura.

A SMS, por exemplo, congrega servidores das categorias profissionais de enfermagem, entre outros. Os índices de absentismo-doença iguais ou superiores a 15 dias nesta secretaria foram de 3,4\% em 2004 e 3,9\% em 2005, índices inferiores aos encontrados na literatura.

Echer et al. (1999) encontraram, entre trabalhadores de enfermagem, uma taxa de absentismo de 6,35\%. Guimarães (2005), em estudo junto a uma instituição hospitalar, encontrou uma Tad de 4,23\%. Em ambos os casos, as autoras trabalharam com índices de licenças (LTS) totais, ou seja, licenças a partir de um dia de afastamento por causas médicas. Se o presente estudo investigasse o total de LTS da referida secretaria, o índice de absentismo-doença poderia ser maior.

\section{Análise das licenças por motivo de doença (LTS)}

As doenças psíquicas e mentais foram as que mais afastaram os servidores, seguidas pelos fatores que influenciam o estado de saúde e o contato com os serviços de saúde e pelas doenças relacionadas ao sistema osteomuscular e do tecido conjuntivo.

Os resultados do estudo coincidem parcialmente com os encontrados na literatura, que indicam, em alguns casos, as doenças respiratórias e osteomusculares como principais responsáveis pelo absentismo-doença (DANATRO, 1997; HAMOUI; SIRIT; BELLORIN, 2005; NOGUEIRA, 1980).

Hamoui, Sirit e Bellorini (2005), em estudo realizado em uma universidade na Venezuela, observaram que as doenças osteomusculares e do tecido conjuntivo, seguidas das doenças relacionadas ao sistema nervoso e aos órgãos do sentido, transtornos mentais, cardiovasculares, traumatismos, envenenamentos, atos cirúrgicos, assim como sinais e sintomas, foram responsáveis pelo aumento dos dias de trabalho perdidos (GUIMARÃES, 2005; RIBEIRO, 2004). Além das doenças respiratórias e osteomusculares, autores relatam que doenças cardiovasculares e do aparelho digestivo também afastam trabalhadores (GUIMARÃES, 2005; RIBEIRO, 2004).

Silveira e Monteiro (2004), em estudo realizado em uma instituição hospitalar, verificaram que as doenças que causaram maior afastamento de trabalhadores foram as cardiovasculares (20\%), as mentais $(15,5 \%)$ e as doenças relacionadas ao sistema musculoesquelético (10\%). As autoras observaram que as doenças de maior prevalência nos principais grupos de doença foram: lombalgia, depressão e hipertensão.

Reis et al. (2003) afirmam, a partir do estudo realizado com profissionais de enfermagem, que as doenças do aparelho respiratório são as que mais afastam os trabalhadores. Em 2003, a cada 1.000 trabalhadores públicos civis da união, 26 ausentaram-se do trabalho, porém, não existem referências em relação ao afasta- 
mento, se este foi em decorrência da atividade profissional ou originado no ambiente de trabalho. As doenças podem agravar-se ou até se originar no ambiente de trabalho. As lesões por esforço repetitivo (LER) foram responsáveis por $17 \%$ dos afastamentos, seguidas pelos casos de depressão e de saúde mental, que superaram o percentual de 5\%. Diabetes e hipertensão também se fizeram presentes como causa dos afastamentos (DOMINGUES JUNIOR, 2005).

Barboza e Soler (2003), em estudo realizado em hospital de ensino, observaram que as licenças-saúde entre o pessoal de enfermagem (enfermeiros, técnicos de enfermagem e auxiliares de enfermagem) ocorreram em função de problemas geniturinários e doenças mal definidas. Entre os atendentes de enfermagem, as moléstias estavam relacionadas aos órgãos do sentido, às doenças infecciosas, parasitárias e às do sistema respiratório.

Das enfermidades que deram origem às LTS no período estudado, algumas estão incluídas no Manual de Procedimentos para os Serviços de Saúde (BRASIL, 2001). Por exemplo, os episódios depressivos são responsáveis por $12,5 \%$ dos afastamentos. É necessário verificar melhor a relação da doença com o local de trabalho e a atividade do servidor.

No presente estudo, foi possível observar um contingente de servidores doentes, afastados, e mais um tanto de servidores sobrecarregados.

Um exemplo a ser seguido pela administração é a política de recursos humanos desenvolvida pelo DMAE, em que são disponibilizados ao servidor um ambulatório médico e um odontológico, farmácia, ambulância e atendimento social (CÂMARA DOS VEREADORES DE PORTO ALEGRE, 2006b).

\section{Referências}

ABRAMIDES, M. B. C.; CABRAL, M. S. R. Regime de acumulação flexível e saúde do trabalhador. São Paulo em Perspectiva, São Paulo, v. 17, n. 1, p. 3-10, mar. 2003. Disponível em: <http://www.scielo.br/scielo. php?script $=$ sci_arttext\&pid $=$ S0102-883920030001000 02\&lng $=$ pt\&nrm $=$ iso $>$. Acesso em: 17 dez. 2007.

BARBOZA, D. B.; SOLER, Z. A. S. G. Afastamentos do trabalho na enfermagem: ocorrências com trabalhadores de um hospital de ensino. Revista Latino-americana de Enfermagem, Ribeirão Preto, v. 11, n. 2, p. 177-183, mar. 2003.

BRASIL. MINISTÉRIO DA SAÚDE. Doenças relacionadas ao trabalho: manual de procedimentos para os serviços de saúde. Brasília: Ministério da Saúde, 2001.
Verificou-se, ainda, que a diferença das proporções é significativa. Considerando uma significância de 5\%, rejeitamos a hipótese de que a diferença entre os anos seja nula (Tcalc $>$ T Tabelado). Logo, existe uma diferença significativa entre as proporções de LTS por mais de 15 dias ao se comparar os anos de 2004 e 2005.

As limitações do estudo estiveram relacionadas, principalmente, à dificuldade de acesso aos dados. A proposta inicial era descrever o perfil epidemiológico de trabalhadores em licença para tratamento de saúde de uma prefeitura, porém, a dificuldade de obtenção de dados primários inviabilizou essa proposta. Diante disso, fomos obrigados a mudar a estratégia e elaboramos um novo projeto, partindo das informações fornecidas pelo gestor. A falta de elementos que poderiam enriquecer o trabalho, como idade, sexo e ocupação dos servidores, entre outros, foi um grande limitador do estudo.

Em suma, os resultados deste estudo não evidenciam uma taxa elevada de absentismo-doença se comparados com os dados de outros estudos. No entanto, tais resultados deverão servir de alerta para este problema que atinge grande parte das organizações, sejam elas públicas ou privadas, e poderão auxiliar os gestores de recursos humanos das diversas secretarias na construção de estratégias de prevenção e controle deste problema, com o intuito de diminuir as taxas encontradas. Conhecer a realidade para intervir: só assim é possível promover e prevenir. Para o êxito no combate ao absentismo-doença entre os servidores públicos municipais, necessita-se, ainda, que os envolvidos se desarmem ideologicamente. O diálogo entre os diferentes sujeitos tem caráter emergencial. A melhoria dos registros junto à GSSM, a implantação de um banco de dados, a informatização plena da gerência e o fomento à pesquisa são alguns elementos que poderão subsidiar propostas de combate ao absentismo-doença na Prefeitura.
CÂMARA DOS VEREADORES DE PORTO ALEGRE. Relatório final da CPI das biometrias. Porto Alegre, 2006a, p. 15.

. Relatório final da CPI das biometrias. Porto Alegre, 2006b. p. 27.

COUTO, H. de A. (Org.). Temas de saúde ocupacional: coletânea dos cadernos ERGO. Belo Horizonte: ERGO, 1987.

DANATRO, D. Ausentismo laboral de causa médica en una institución pública. Revista Médica del Uruguay, Montevidéu, v. 13, n. 2, p. 101-109, ago. 1997.

DEJOURS, C. Por um novo conceito de saúde. Revista Brasileira de Saúde Ocupacional, São Paulo, v. 14, n. 54, p. 7-11, 1986.

DOMINGUES JUNIOR, L. R. P. O Processo saúde -doença no serviço público e suas conseqüências ao 
Estado, ao cidadão e ao servidor. In: CONFERÊNCIA NACIONAL DE SAÚDE DO TRABALHADORTEXTOS DE APOIO, 3., 2005, Brasília. Coletânea №1. Brasília: Ministério da Saúde, 2005. p. 54-55.

DURAND, J. C.; BELTRÃO, R. E. V. Recensear funcionários públicos: uma necessidade. Revista do Serviço Público, Brasília, v. 118, n. 2, p. 185-198, 1994.

ECHER, I. C. et al. Estudo do absenteísmo como variável no planejamento de recursos humanos em enfermagem. Revista Gaúcha de Enfermagem, Porto Alegre, v. 20, n. 2, p. 65-76, jul.1999.

FARIA, A. C.; BARBOZA, D. B.; DOMINGOS, N. A. M. Absenteísmo por transtornos mentais na enfermagem no período de 1995 a 2004. Arquivos Ciência e Saúde, São José do Rio Preto, v. 12, n. 1, p. 14-20, jan./mar. 2005.

FRANÇA, B. H. Funcionário público: trabalhador como os outros? Revista do Serviço Público, Brasília, v. 118, n. 2, p. 199-212, jul./ago. 1994.

FUNDACIÓN EUROPEA PARA LA MEJORA DE LAS CONDICIONES DE VIDA Y TRABAJO. A prevenção do absentismo no trabalho - sinopse da investigação. Luxemburgo: Serviço das Publicações Oficiais das Comunidades Européias, 1997. Disponível em: <http://www.eurofound.europa.eu/pubdocs/1997/15/ pt/1/ef9715pt.pdf>. Acesso em: 05 mai. 2007.

GOMERO CUADRA, R.; LLAP YESAN, C. Absentismo laboral de origem médico en el Hospital Toquepala en el 2001. Revista Médica Herediana, Lima, v. 15, n. 2, p. 95-100, abr./jun. 2004.

GUIMARÃES, R. S. O. O absenteísmo entre os servidores civis de um Hospital Militar. 2005. $83 \mathrm{f}$. Dissertação (Mestrado em Saúde Pública)-Fundação Oswaldo Cruz, Rio de Janeiro, 2005.

HAMOUI, Y.; SIRIT, Y.; BELLORIN, M. Absentismo laboral del personal administrativo de una universidad pública Venezolana, 2000-2002. Revista Salud de los Trabajadores, Maracay, v. 13, n. 2, p. 107-118, jul./dic. 2005.

HURREL, J. et al. (orgs.) Salud Social. In: ORGANIZACIÓN MUNDIAL DEL TRABAJO. Enciclopedia de Salud y Seguridad en el Trabajo. Madrid: Ministério de Trabajo y Asuntos Sociales, 1998. p. 2-12 .

INSTITUTO BRASILEIRO DE GEOGRAFIA E ESTATÍSTICA. Pesquisa Nacional por Amostra de Domicilio - Síntese de Indicadores. Rio de Janeiro: IBGE, 2007.

ISOSAKI, M. Absenteísmo entre trabalhadores de serviços de nutrição e dietética de dois hospitais em São Paulo. Revista Brasileira de Saúde Ocupacional, São Paulo, v. 28, n. 107/108, p. 107-118, 2003.
MALLADA, F. J. R. A gestão do absentismo trabalhista nas empresas espanholas. Universidade de Alcalá de Henares, 2004. Disponível em: < http://kplus.cosmo. com.br/materia.asp?co=131\&rv=Direito $>$. Acesso em: 23 jul. 2007.

MARTINS, R. J. et al. Absenteísmo por motivos odontológico e médico nos serviços público e privado. Revista Brasileira de Saúde Ocupacional, São Paulo, v. 30, n. 111, p. 9-15, 2005.

MENDES, R. O impacto dos efeitos da ocupação sobre a saúde de trabalhadores. I. Morbidade. Revista de Saúde Pública, São Paulo, v. 22, n. 4, p. 311-326, ago. 1988.

NOGUEIRA, D. P. Absenteísmo-doença: aspectos epidemiológicos. 1980. 264 f. Tese (Livre Docência em Saúde Pública)-Faculdade de Saúde Pública, Universidade de São Paulo, São Paulo, 1980.

PORTO ALEGRE. Lei Complementar no 133, de 31 de dezembro de 1985. Estatuto dos Funcionários Públicos do Município de Porto Alegre, Porto Alegre, RS, $31 \mathrm{dez.}$ 1985.

PREFEITURA MUNICIPAL DE PORTO ALEGRE. Anuário Estatístico - 2005. Porto Alegre: Prefeitura Municipal de Porto Alegre / Gabinete de Programação Orçamentária / Gerência de Estatística, 2006.

QUICK, T. C.; LAPERTOSA, J. B. Análise do absentismo em usina siderúrgica. Revista Brasileira de Saúde Ocupacional, São Paulo, v. 10, n. 40, p. 6267, out./dez. 1982.

REIS, R. J. et al. Fatores relacionados ao absentismo por doença em profissionais de enfermagem. Revista de Saúde Pública, São Paulo, v. 37, n. 5, p. 616-623, out. 2003.

RIBEIRO, L. F. Absenteísmo no setor bancário: análise de um programa de intervenção. 2004. 85 f. Dissertação (Mestrado em Saúde Pública)-Universidade Federal da Bahia, Salvador, 2004.

SILVA, D. M. P. P.; MARZIALE, M. H. P. Absenteísmo de trabalhadores de enfermagem em um hospital universitário. Revista Latino-americana de Enfermagem, Ribeirão Preto, v. 8, n. 5, p. 44-51, out. 2000.

SILVEIRA, D. B. B.; MONTEIRO, S. M. Morbidade entre trabalhadores de uma instituição de saúde. Revista Saúde em Debate, Rio de Janeiro, v. 28, n. 68, p. 206-211, set./dez. 2004.

TENÓRIO, L. R.; JORDANO, A. Absenteísmo-doença em construção civil. In: ANAIS DO CONGRESSO NACIONAL DE PREVENÇÃO DE ACIDENTES DO TRABALHO, 18., 1979, Salvador. Anais... São Paulo: Fundacentro, 1980. p. 21-25. 